\title{
DOSE RESPONSE OF XYLITOL AND SORBITOL FOR EPR RETROSPECTIVE DOSIMETRY WITH APPLICATIONS TO CHEWING GUM.
}

Axel Israelsson, Håkan Gustafsson and Eva Lund

\section{Linköping University Post Print}

N.B.: When citing this work, cite the original article.

This is a pre-copy-editing, author-produced PDF of an article accepted for publication in Radiation Protection Dosimetry following peer review. The definitive publisher-authenticated version:

Axel Israelsson, Håkan Gustafsson and Eva Lund, DOSE RESPONSE OF XYLITOL AND SORBITOL FOR EPR RETROSPECTIVE DOSIMETRY WITH APPLICATIONS TO CHEWING GUM., 2012, Radiation Protection Dosimetry. is availabel online at: http://dx.doi.org/10.1093/rpd/ncs174 Copyright: Oxford University Press (OUP): Policy B http://www.oxfordjournals.org/

Postprint available at: Linköping University Electronic Press http://urn.kb.se/resolve?urn=urn:nbn:se:liu:diva-85528 


\title{
DOSE RESPONSE OF XYLITOL AND SORBITOL FOR EPR RETROSPECTIVE DOSIMETRY WITH APPLICATIONS TO CHEWING GUM
}

\author{
A. Israelsson*1, H. Gustafsson ${ }^{1}$ and E. Lund ${ }^{1}$
}

Radiation Physics, Department of Medical and Health Sciences, Linköping University, Linköping, Sweden

The purpose of this investigation was to study the radiation-induced EPR signal in sweeteners xylitol and sorbitol for use in retrospective dosimetry. For both sweeteners and chewing gum, the signal changed in an interval of 1-84 days after irradiation with minimal changes 4-8 days after. A dependence on storage conditions was noticed and the exposure of the samples to light and humidity was therefore minimized. Both the xylitol and sorbitol signals showed linearity with dose in the measured dose interval, 0-20 Gy. The dose response measurements for the chewing gum resulted in a decision threshold of $0.38 \mathrm{~Gy}$ and a detection limit of $0.78 \mathrm{~Gy}$. A blind test illustrated the possibility of using chewing gums as a retrospective dosemeter with an uncertainty in the dose determination of 0.17 Gy (1 SD).

Keywords:

EPR, ESR, retrospective dosimetry, radiation, xylitol, sorbitol

Running head: Dose response of xylitol and sorbitol

${ }^{*}$ Corresponding author:

Email: axel.israelsson@liu.se,

Telephone number: +46 10 1033452, Fax: +46 011032895

Radiation Physics

Dept. of Medical and Health Sciences

Linköping University

SE 58185 Linköping

Sweden 


\section{INTRODUCTION}

When radiation accidents occur, it is of great importance to assess the radiation doses experienced by the exposed individuals. Accidents and malevolent attacks causing nuclear emergencies may involve large amounts of people. Many people may be exposed, and many others will worry that they have been exposed to radiation. It is important to quickly determine the doses delivered to individuals. This investigation is part of the effort by the Swedish Radiation Safety Authority to find quick retrospective methods. Within one week after an accident updates on the determined dose will be helpful to guide the medical treatment. Individuals without combined injury and sub clinical exposures, i.e. less than $1.5 \mathrm{~Gy}$, can be followed as outpatients ${ }^{(1)}$. Dose determinations are also important for long-term individual risk assessments and for dose reconstructions.

Common techniques used for retrospective dosimetry are electron paramagnetic resonance (EPR) ${ }^{(2)}$, thermoluminescent dosimetry (TLD) ${ }^{(3)}$, optically stimulated luminescence (OSL) ${ }^{(4)}$ and various forms of biodosimetry ${ }^{(5)}$. The techniques are often used together at radiation events, as they complement each other well. The best choice is dependent on the available substances that are suitable for dosimetry.

EPR dosimetry is the most widely used technique for retrospective dose determinations. In contrast to biodosimetry, EPR dosimetry is mainly used to determine doses of external radiation; both whole and partial body exposures.

EPR dosimetry is based on the quantification of radiation-induced paramagnetic centres, which are proportional to the absorbed radiation energy. Since the signal is preserved during read out, the samples may be repeatedly measured. Dose response is generally linear in a broad dose interval and the calibration can be performed using either the calibration curve method or by the additive dose method, as described in an IAEA report for tooth enamel dosimetry ${ }^{(6)}$. In the calibration method, reference samples are irradiated and measured to obtain their dose responses, while in the additive dose method, the actual sample is incrementally irradiated with well-known doses and measured. When using reference samples, it is important that they are identical or very similar to the actual sample.

In body materials like tooth enamel, bone and nails, long-lived free radicals are created by ionizing radiation, and therefore, these materials can serve as dosemeters for analysis with EPR spectroscopy ${ }^{(7-9)}$. Other materials found in the pockets of individuals such as sugar ${ }^{(10)}$, watch glass ${ }^{(1)}$ and mobile phone LCD glass ${ }^{(12)}$ are also suitable. The usefulness of a particular material as a dosemeter can be evaluated by investigating its pertinent properties, in addition to being ubiquitous: the material should have high radiation-induced signal specificity, a low background signal, a low UV-induced signal, a low detection limit, linearity of the signal with dose at low doses and high signal $^{(2)}$.

Sucrose has proven to be suitable for retrospective dose determinations after several radiological accidents (13-16). Following mass casualties such as terrorist attacks, the chances of finding pure sugar close to an exposed individual might not be high. Rather, it may be present as an ingredient in confectionery, chewing gums or medicines carried by the victims. Nowadays, however, sweeteners such as xylitol, sorbitol and aspartame are often used instead of sugar as sweetening agents in those products. It is, therefore, of interest to study the radiation-induced EPR signal in sweeteners such as xylitol, sorbitol and aspartame and investigate the possibility of using these signals for accidental and retrospective dosimetry.

A few EPR studies on these sweeteners have been published. Hervé et al. ${ }^{(17)}$ have reported studies of several sweeteners, including sorbitol, in a mixed gamma and neutron field including measurements with respect to light sensitivity, time dependence and linearity ${ }^{(18)}$. Sorbitol as an ingredient in medical tablets was analyzed for retrospective dose determinations after an accident in Norway in $1982^{(15)}$. Aspartamebased sweeteners have also been studied ${ }^{(19)}$. Radical studies have been performed on single crystals of xylitol and sorbitol irradiated and measured at low temperatures of approximately $4 \mathrm{~K}^{(20)}$.

The spectra from irradiated carbohydrates are often rather complex. Several peaks corresponding to different radicals overlap each other, which complicates the interpretation of the spectra as illustrated for sucrose by Vanheylewyn et $\mathrm{al}^{(21)}$. Radicals may have different microwave saturation characteristics, and their abundance may also change over time. The signal evolution with time (i.e., signal stability) is important to evaluate because a change of intensity over time may lead to misinterpretations of the radiation-induced signal. Shortly after irradiation (1-15 days), the signal stability is of special interest because an important area of application for sweeteners as retrospective dosemeters is to determine doses as soon as possible after irradiation.

To the best of our knowledge, xylitol has not previously been studied for use as a radiation dosemeter. The main purpose of the present study is 
therefore to investigate the radiation-induced signal in both xylitol and sorbitol to allow for accurate dose determinations using sweets and chewing gums that contain these sweeteners.

\section{MATERIALS AND METHODS}

In the present work, the EPR spectra of irradiated sorbitol and xylitol were carefully examined with the aim of identifying the most suitable peaks for dose acquisitions, including studies of the spectral changes with time after irradiation and the EPR signal dependence on storage conditions. An evaluation of the optimum read-out microwave power for dose determinations was also performed, and the linearity of the signal intensity of the chosen peaks with absorbed dose was studied. A method of determining the dose delivered to irradiated chewing gums is also presented and verified with blind test samples imitating samples from a real accident.

Each experiment followed roughly the same scheme of sample preparation, irradiation, EPR measurement and data analysis. The general method is described below and each phase is summarized in Table 1 .

\section{General method}

\section{Sample preparation}

Crystalline xylitol and sorbitol, purchased from SigmaAldrich, and coating from the chewing gum Dental V6 (Cadbury Sweden AB) were pressed into cylindrical tablets in a manual pellet press. No binding material was needed and no additional signal from pressing was observed in the following EPR measurements. Only the coating of the chewing gum was used because the sweetening is mainly located there; a gum base mixture constitutes the interior. The coating was mechanically separated from the gum base. From each chewing gum typically $150 \mathrm{mg}$ coating can be used. The tablets had a diameter of $5 \mathrm{~mm}$ and a height of either 4.5 or $9 \mathrm{~mm}$. During storage, all tablets were kept in sealed, lightproof containers with the exception for three tablets of each xylitol and sorbitol, which for the storage condition experiment were kept in open air exposed to daylight.

\section{Irradiation}

The irradiations were performed at room temperature with a $6 \mathrm{MV}$ photon beam from a Varian Clinac 600 $\mathrm{C} / \mathrm{D}$ accelerator. The samples were placed at a $4 \mathrm{~cm}$ depth in a PMMA phantom with a height of $17 \mathrm{~cm}$ and an area of $20 \mathrm{~cm} \times 20 \mathrm{~cm}$. The source-to-surface distance was $100 \mathrm{~cm}$, and the radiation field size was
$10 \mathrm{~cm} \times 10 \mathrm{~cm}$. The absorbed doses were approximated as absorbed dose in water based on the gantry detector recordings when no precise doses were needed.

\section{Measurements}

The measurements were performed with a BRUKER EleXsys E580 EPR spectrometer operating at X-band equipped with an ER 4102ST standard cavity for spectral investigations and a JEOL JES FR30EX Free Radical Monitor for signal intensity measurements. The tablets were placed in a quartz sample tube (Suprasil, Heraeus Quarzglas GmbH \& Co. KG) with a diameter of $5 \mathrm{~mm}$, and a $\mathrm{Mn}^{2+/} \mathrm{MgO}$ reference was used as a spectrometer field stability control.

\section{Analysis}

The acquired spectra were corrected for the base line slope by the subtraction of a linearly fitted base line. The field sweep had a width of $10 \mathrm{mT}$ and was centered between the third $(\mathrm{g}=2.034)$ and fourth $(\mathrm{g}=$ 1.981) $\mathrm{Mn}^{2+} / \mathrm{MgO}$ lines. The third line served as the reference, as the spectra were normalized to the magnetic field value of that line. In some experiments, the spectra were thereafter smoothed for the purpose of noise reduction. The smoothing was performed by averaging each point in the spectrum over the nearest $\mathrm{N}$ points, with $\mathrm{N} \approx 3-6 \%$ of the total number of measurement points. The dose response signal was then determined by reading out a peak-to-peak amplitude from the spectrum. Data analysis was performed in MATLAB (The MathWorks Inc.).

\section{Dose response measurement and verification of the method with a blind test}

Ten V6 chewing gum pieces from different manufacturing batches had their coating pressed into one tablet each. The background signal was measured 20 times for each tablet. Five of the ten tablets were then irradiated to 4.0 Gy and were measured again 4-6 days after irradiation, 20 times each. These measurements of non-irradiated and irradiated samples then served as calibration curve. Four additional samples were irradiated with doses unknown to the experimenter. Two of these were irradiated as whole pieces of chewing gum and two as tablets. All samples were irradiated at a depth of $8.5 \mathrm{~cm}$ in a PMMA phantom with a surface area of $20 \mathrm{~cm} \times 20 \mathrm{~cm}$. To assure full backscatter, $20 \mathrm{~cm}$ of PMMA was also placed behind the samples. The Source Sample Distance (SSD) was $100 \mathrm{~cm}$, and the field size was 12 $\mathrm{cm} \times 12 \mathrm{~cm}$. For accurate dose determinations, a Farmer NE 2571 ionization chamber traceable to BIPM was placed at the same depth and distance from the 
field center to simultaneously determine the absorbed dose in water. A calibration curve was obtained with the least-squares fit method, and the blind test doses were deduced using this curve. The decision threshold and detection limit of the dose response measurement were deduced in accordance with ISO $11929^{(22)}$ and with guidance from Currie ${ }^{(23)}$. The uncertainty analysis is included in the Appendix.

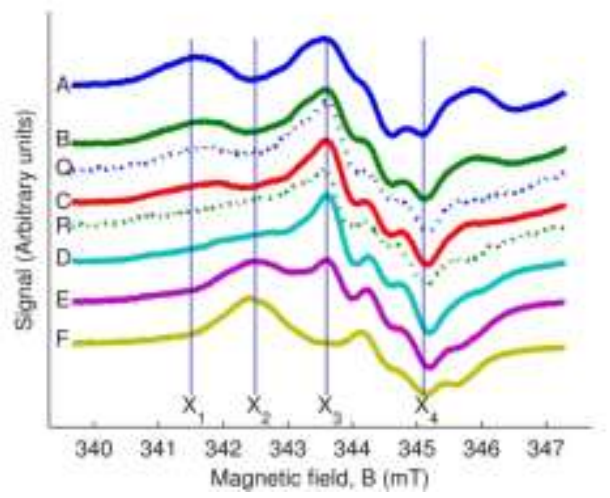

Figure 1. EPR spectra of xylitol and V6 irradiated to 50 Gy. The xylitol spectra were measured $A=1$ day, $B=4$ days, $\mathrm{C}=8$ days, $\mathrm{D}=16$ days, $\mathrm{E}=36$ days and $\mathrm{F}=84$ days after irradiation, while the dashed V6 spectra were measured $\mathrm{Q}=4$ days and $\mathrm{R}=8$ days after irradiation. For visualisation reasons, all spectra have been separated from each other, and due to their lower signal, the two V6 spectra have been arbitrarily multiplied with 1.7 . The microwave power used was $1 \mathrm{~mW}$. The peaks $\mathrm{X}_{1}, \mathrm{X}_{2}, \mathrm{X}_{3}$ and $\mathrm{X}_{4}$ were used for saturation measurements. All spectra were normalized to a $\mathrm{Mn} 2+/ \mathrm{MgO}$ reference.

\section{RESULTS}

\section{Spectrum variation with time}

Several peaks were detected at different magnetic field values. Signal dependence with time after irradiation was apparent, as the amplitudes of some peaks changed between measurements. At one day after exposure, the xylitol measurements showed pronounced peaks: $X_{1}$ and $X_{3}$ (see figure 1). The $X_{1}$ peak thereafter decreased after one day, while the $X_{3}$ peak slightly increased with time up to 16 days, when it began to decrease. A peak $\mathrm{X}_{2}$, appeared at 36 days and increased with time until at least 84 days after irradiation. The magnitude of the negative peak, $\mathrm{X}_{4}$ initially increased until 16 days after exposure, after which it decreased; this behavior resembles that of the $\mathrm{X}_{3}$ peak.

The sorbitol spectrum changed less with time after irradiation than the xylitol spectrum (see figure 2). One peak, $S_{1}$ was found to be rather stable through all measurements, while $S_{2}$ increased up to 36 days. The magnitude of a third peak, the negative peak $\mathrm{S}_{3}$, decreased gradually with time after exposure. These measurements indicate that $S_{1}$ is the most stable signal and therefore best suited for dose-response determinations.

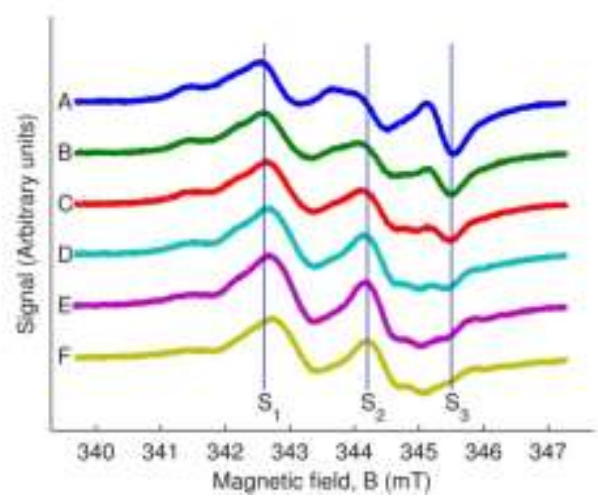

Figure 2. EPR spectra of sorbitol irradiated to $50 \mathrm{~Gy}$, measured $\mathrm{A}=1$ day, $\mathrm{B}=4$ days, $\mathrm{C}=8$ days, $\mathrm{D}=16$ days, $\mathrm{E}=$ 36 days and $\mathrm{F}=84$ days after irradiation. For visualisation reasons, all spectra have been separated from each other. The microwave power used was $1 \mathrm{~mW}$. The peaks $\mathrm{S}_{1}, \mathrm{~S}_{2}$ and $\mathrm{S}_{3}$ were used for saturation measurements. All spectra were normalized to a $\mathrm{Mn}^{2+} / \mathrm{MgO}$ reference.

An identical study was performed for the V6 chewing gum. The resulting spectra showed a clear similarity to those of xylitol. This was expected because the chewing gum coating has a high relative abundance of xylitol. The V6 spectra of the most relevant time periods, 4 and 8 days after irradiation, are displayed as dashed lines alongside the xylitol spectra in figure 1. The spectrum of unirradiated V6 did not display any peaks distinguishable from noise.

\section{Power saturation}

The power saturation measurements were analyzed for peaks $\mathrm{X}_{1-4}$ for xylitol and $\mathrm{S}_{1-3}$ for sorbitol, and the results are shown in figure 3 . The signal intensity of each investigated peak was measured with respect to the microwave power at the time after irradiation when that specific peak was most prominent. For xylitol, $\mathrm{X}_{1}$ and $\mathrm{X}_{2}$ were measured 1 and 84 days after irradiation, respectively, while $X_{3}$ and $X_{4}$ were measured 16 days after irradiation. For sorbitol, $S_{1}$ and $S_{3}$ were measured 1 day after irradiation, and $S_{2}$ was measured 16 days after irradiation. The negative peaks $\mathrm{X}_{4}$ and $\mathrm{S}_{3}$ are represented by their absolute values. From the figure, it can be seen that the peaks respond differently to increased power. For xylitol, $\mathrm{X}_{2-4}$ are not saturated even at a microwave power of $100 \mathrm{~mW}$, while $\mathrm{X}_{1}$ is saturated at about $5 \mathrm{~mW}$. The sorbitol peaks $\mathrm{S}_{1}$ and $\mathrm{S}_{3}$ 
saturate at about 10 and $30 \mathrm{~mW}$ respectively, while $S_{2}$ saturates at $3 \mathrm{~mW}$.
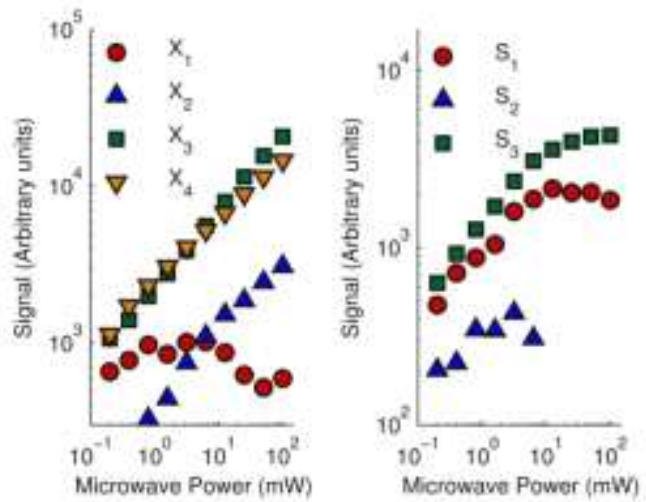

Figure 3. Left-hand plot: Saturation curves of xylitol for the peaks $X_{1}, X_{2}, X_{3}$ and $X_{4}$ (see figure 1). Right-hand plot: Saturation curves of sorbitol for the peaks $S_{1}, S_{2}$ and $S_{3}$ (see figure 2). Each curve was obtained at the time after irradiation when the corresponding peak was most pronounced; $X_{1}, S_{1}$ and $S_{3}$ were measured 1 day after irradiation, $X_{3}, X_{4}$ and $S_{2}$ were measured 16 days after, and $X_{2}$ was measured 84 days after irradiation.

\section{Effects of storage conditions}

The storage condition test showed a difference for xylitol but not for sorbitol between the spectra obtained from the tablets exposed openly and from those that were kept in a dark, sealed container after irradiation; see figure 4 . The $X_{2}$ peak was slightly higher for the exposed samples, while the $\mathrm{X}_{3}$ peak was lower.

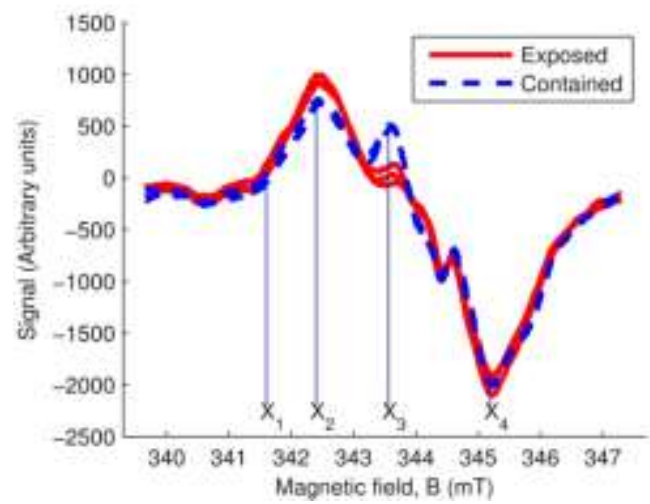

Figure 4. Xylitol spectra showing different curve shapes depending on storage conditions. The solid curves indicate tablets that were exposed to daylight in open air, while the dashed ones indicate tablets that were kept in a lightproof, sealed container. Three spectra of each kind are plotted. $\mathrm{X}_{1-4}$ mark the peaks that were followed in the signal-evolutionwith-time and power-saturation experiments.

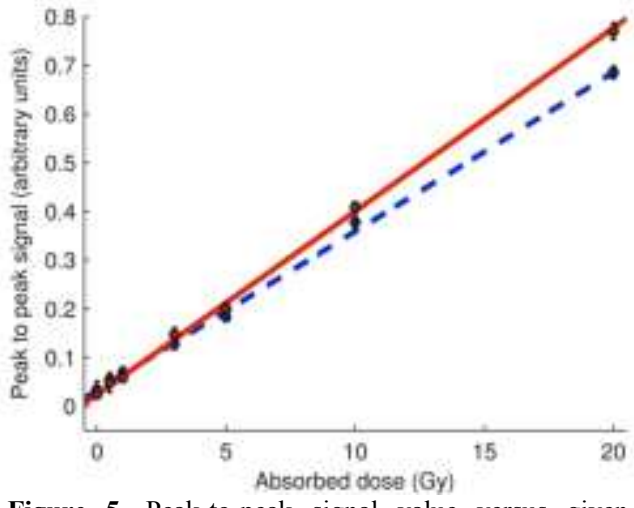

Figure 5. Peak-to-peak signal value versus given dose, measured on peaks $X_{3}$ and $X_{4}$ for xylitol and $S_{1}$ and $S_{3}$ for sorbitol. The solid line represents xylitol, and the dashed line represents sorbitol. The error bars corresponds to \pm 1 standard deviation. For xylitol, the squared correlation coefficient is $R^{2}=1.00$, the calibration factor $a=(37 \pm 1) \cdot 10^{-3} \mathrm{~Gy}^{-1}$ and the peak-to-peak signal at a zero dose $P_{0}=(23 \pm 5)$. $10^{-3}$. For sorbitol, $R^{2}=0.99, a=(32 \pm 1) \cdot 10^{-3} \mathrm{~Gy}^{-1}$ and $P_{0}=(31 \pm 5) \cdot 10^{-3}$. The spectra were normalized to the $\mathrm{Mn}^{2+} / \mathrm{MgO}$ reference line intensity.

\section{Linearity of the dose response}

The linearity of the peak-to-peak signal versus dose for xylitol and sorbitol is shown in figure 5 . The peak-topeak values used were obtained from the $\mathrm{X}_{3}$ and $\mathrm{X}_{4}$ peaks for xylitol and from the $S_{1}$ and $S_{3}$ peaks for sorbitol. The square of the correlation coefficients were determined to be $R^{2}=1.00$ for xylitol and $R^{2}=0.99$ for sorbitol in the interval 0-20 Gy.

\section{Dose response measurement and verification of the method with a blind test}

The chewing gum calibration curve is displayed in the left-hand panel of figure 6 . The measured peak-to-peak values are obtained from the V6 peaks that correspond to $\mathrm{X} 3$ and $\mathrm{X} 4$ in figure 1 . The peak-to-peak values from the ten background samples were measured to be $P_{0}=82 \pm 9$ a.u., and marks the intercept with the yaxis. The samples irradiated to $4 \mathrm{~Gy}$ were measured to be $P_{1}=278 \pm 6$ a.u., which yields a slope value, i.e., calibration factor, of $a=48 \pm 4 \mathrm{~Gy}^{-1}$. The decision threshold and detection limit were calculated to be $D^{*}=0.38$ Gy and $D^{\#}=0.78 \mathrm{~Gy}$, respectively (see Appendix).

The results from the blind dose determination are displayed in table 2 and in the residual plot in the righthand panel of figure 6. Samples $\mathrm{A}$ and $\mathrm{C}$ were irradiated as tablets similar to the calibration samples, while samples B and D were irradiated as whole pieces 
of chewing gum before being pressed into tablets. The samples were given the following doses: $\mathrm{A}_{\mathrm{g}}=0.85 \mathrm{~Gy}$, $\mathrm{B}_{\mathrm{g}}=0.88 \mathrm{~Gy}, \mathrm{C}_{\mathrm{g}}=2.97 \mathrm{~Gy}$ and $\mathrm{D}_{\mathrm{g}}=3.05$. The doses were then experimentally measured to be $A_{m}=0.74 \pm$ $0.17 \mathrm{~Gy}, \mathrm{~B}_{\mathrm{m}}=0.95 \pm 0.17 \mathrm{~Gy}, \mathrm{C}_{\mathrm{m}}=2.81 \pm 0.15 \mathrm{~Gy}$ and $\mathrm{D}_{\mathrm{m}}=3.15 \pm 0.14 \mathrm{~Gy}$, respectively, where the uncertainties are given as one standard deviation (SD). All determinations except one fall within the 1 SD uncertainty of the true value, and the exception falls within 2 SD.
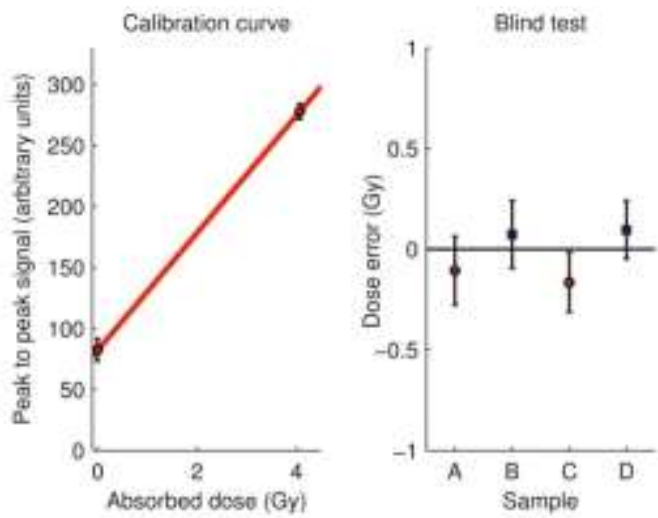

Figure 6. Left-hand plot: The dose response calibration curve of V6 chewing gum. The slope value, i.e., the calibration factor, is $a=48 \pm 4 \mathrm{~Gy}^{-1}$, and the zero intercept, i.e., the background, is $P_{0}=82 \pm 9$. Right-hand plot: Residual plot of the blind test samples. Given doses were ' $\mathrm{A}$ ' $=0.85 \mathrm{~Gy}$, ' $\mathrm{B}$ ' = $0.88 \mathrm{~Gy},{ }^{\prime} \mathrm{C}$ ' $=2.97 \mathrm{~Gy}$ and ' $\mathrm{D}$ ' $=3.05 \mathrm{~Gy}$. The uncertainties are given as 1 standard deviation.

Because the calibration measurements of the irradiated samples were performed over three days, the signal stability within this interval was checked. When the 10 background samples and the 5 irradiated samples were measured 20 times during a period of 3 days, no difference in signal intensity was registered from day 1 to day 3 .

The measurement uncertainties of the blind test samples are given in table 2. Because the sample irradiation and measurements were performed under the same laboratory conditions as the calibration, there was no extra uncertainty due to differences in the calibration and blind-test conditions. Differences in time after irradiation, storage, irradiation temperature and radiation quality were all negligible, as described in the Appendix.

\section{DISCUSSION}

Xylitol and sorbitol are, together with aspartame, common sweetening agents often used in place of natural sugar in chewing gums. A few preliminary experiments on pure irradiated aspartame conducted in our laboratory did not reveal any detectable EPR signal, in contrast to previously published results ${ }^{(19)}$ and ${ }^{(24)}$

The spectral shapes vary with time after irradiation for both sweeteners, as seen in figures 1 and 2 . Three peaks for xylitol and two peaks for sorbitol are seen to vary considerably between 1 and 84 days. The spectrum variations in the time period 4-8 days after irradiation were minimal for both sorbitol and xylitol as well as for V6 chewing gum. It would have been beneficial to have stable signals over longer time periods. However, in an acute real scenario, information on when the irradiation took place is often readily available, which makes it possible to correct for any time dependence if the signal is well characterized. This can ideally be performed by making the calibration measurements in the same time period after irradiation as the unknown sample or, alternately, by multiplication by a correction factor determined in advance. In case the time of irradiation in an acute scenario is not known, it may be possible to deduce it from the spectral shape. The spectral changes with time may be caused by transformations of the radicals or the surroundings in the samples. The differing saturation behavior between peaks (see figure 3) is a further indication that there is more than one radical present. To clarify the appearance of the radicals present, a more detailed investigation is needed, which is beyond the scope of this article.

The peaks $\mathrm{X}_{2-4}, \mathrm{~S}_{1}$ and $\mathrm{S}_{3}$ showed favorable saturation behavior, as they offer the possibility of measurement at higher read-out microwave powers, allowing for a better signal-to-noise ratio without distorting the signal. The peaks $\mathrm{X}_{3}$ and $\mathrm{X}_{4}$ for xylitol and $\mathrm{S}_{1}$ and $\mathrm{S}_{3}$ for sorbitol were chosen as doseresponsive peak-to-peak values because of their high intensity at the measured time after irradiation. In the present investigation however the microwave power was kept well below the saturation to ensure high signal reproducibility.

When xylitol was exposed to daylight in open air for an extended period of time, 69 days, a spectral change was seen similar to that appearing after a longer time interval in the dark. The $\mathrm{X}_{2}$ peak are in both cases enhanced in comparison with the $\mathrm{X}_{3}$ peak. Whether the cause of the more rapid spectral change was light, moisture or something else is not known, and the reason behind the effect needs to be clarified. This experiment shows that it is important to keep samples containing xylitol sealed and in the dark in acute scenarios. Chewing gums are expected to be carried under these conditions in most cases.

The dose response of chewing gum was chosen, in the present work, to be obtained through the calibration curve method. The alternative additive-dose method has the advantage that no reference samples are needed. However, that method requires that the background signal can be neglected; at least, be small compared 
with the irradiation signal. For the investigated chewing gum, the background signal corresponds to a dose of $1.7 \mathrm{~Gy}$, which cannot be neglected. The calibration curve method requires reference samples that are identical or very similar to the actual samples. The similarity was assured by obtaining the calibration curve from samples taken from different manufacturing batches. An important advantage of the calibration curve method is that neither the calibration samples nor the sample for which the dose is to be determined must be exposed to radiation more than once. This is of importance when the samples, like those in the present case, show spectral dependence with time, particularly soon after irradiation.

The calibration curve was constructed from two dose end points, as recommended by Nagy ${ }^{(25)}$. These were chosen to be the signal of non irradiated samples and of a dose well above the dose to be determined. The linearity between these two points was assured in separate measurements for both xylitol and sorbitol. A similar check for the chewing gum was not necessary because its dose-responsive components showed a linear dose response.

Chewing gums are thought to be carried by many people, either in their pockets or in a bag. Scenarios for which they are likely to be best suited for retrospective dosimetry include "dirty bomb" terrorist attacks and accidents with so-called orphan sources, wherein radionuclides are dispersed and many people fear exposure to radiation. The probability of these scenarios is not negligible, as orphan sources already have figured in accidents. ${ }^{(13)}$

The analysis of irradiated chewing gums in an acute situation can begin as soon as the samples arrive at the EPR laboratory. With the accuracy reported in this article, the analysis of a sample could be completed within three days. With the present method, the capacity of our laboratory allows for the analysis of 6 additional samples per day. However, the time needed will depend on how soon after irradiation the acute samples arrive at the laboratory. It is reasonable to think that the dosimetry of samples from an incident could be finalized within 5 days. The total elapsed time could be reduced substantially, through fewer and shorter measurements, if a lower dose-determination accuracy were accepted.

Once the dose delivered to a piece of chewing gum carried by an exposed individual has been determined, further information, such as type of exposure, is needed to estimate the whole-body dose to the individual and to make a dose reconstruction at the site of exposure. Dose assessments of persons not carrying chewing gum can also be made. Surveillance cameras in public places can be a source of information on where individuals were at the time of exposure.

The decision threshold of 0.38 Gy obtained for V6 chewing gum shows that the proposed method may be used as a retrospective dosemeter. For dose assessments above $0.5 \mathrm{~Gy}$, the present method works well, as shown by the good accordance between the irradiated and measured doses in the blind test.

\section{CONCLUSION}

In the present study, the complex EPR spectra of irradiated sweeteners xylitol and sorbitol were examined. The spectral change with time after irradiation is well characterized and the changes are minimal in the time period 4-8 days. Xylitol and sorbitol should be kept sealed and in the dark in order not to induce signal changes of the acquired spectra. A method for dose determination in irradiated chewing gum was tested successfully in a blind test with uncertainties of 0.3 Gy (2 SD). Chewing gum containing xylitol or sorbitol could be used for retrospective dose determination with a detection limit of 0.8 Gy. This dose level is sufficient for making determinations within a week after irradiation, but should optimally be lower for the purpose of triage and to make risk assessments following nuclear incidents. The discovered possibility to increase the microwave power, may serve to lower the detection limit.

\section{APPENDIX: UNCERTAINTY ANALYSIS}

The estimator of the measurand, $\mathrm{P}$, represents the massnormalized peak-to-peak signal and is given by the linear relation

$$
P=a D+P_{0} .
$$

Here, $\mathrm{D}$ is the absorbed dose to the sample in water, $\mathrm{P}_{0}$ is the peak-to-peak signal at a zero dose and a is the calibration factor.

The quality of the signal response is described by its decision threshold $p^{*}$ and its detection limit $p^{\#}$. If the uncertainty of the estimator $\mathrm{P}$ as a function of the true value $\tilde{p}$ is denoted by $\tilde{u}(\tilde{p})$ and $p_{j}$ is the value from different measurements $(j=0,1,2, \ldots)$, then

$$
p^{*}=k_{1-\alpha} \tilde{u}\left(P_{0}\right)
$$

and

$$
p^{\#}=p^{*}+k_{1-\alpha} \tilde{u}\left(p^{\#}\right),
$$

where $\mathrm{k}_{1-\alpha}$ is the quantile of the standardized normal distribution for the probability $1-\alpha$.

If $\tilde{\mathrm{u}}\left(\mathrm{P}_{0}\right)=\mathrm{u}\left(\mathrm{p}_{0}\right), \mathrm{p}_{1}>0$ and $\mathrm{u}\left(\mathrm{p}_{1}\right)$ is known, the uncertainty can be obtained from

$$
\tilde{u}^{2}(\tilde{p})=\tilde{u}^{2}(0)\left(1-\tilde{p} / p_{1}\right)+\tilde{u}^{2}\left(p_{1}\right) \tilde{p} / p_{1} .
$$


The corresponding decision threshold and detection limit in units of the dose, $D^{*}$ and $D^{\#}$, are determined by $D^{*}=p^{*} / a$ and $D^{\#}=p^{\#} / a$, where the calibration factor a from equation (1) is determined by linear regression. $p^{*}$ and $p^{\#}$ are based on statistical methods as Type A evaluations of uncertainty. The uncertainty of the calibration factor, $\mathrm{u}(a)$, will, however, systematically influence the decision threshold and detection limit ${ }^{(23)}$. As a Type $B$ evaluation of uncertainty, it therefore cannot simply be compounded in the decision threshold or detection limit. $D^{*}$ and $D^{\#}$ should then be characterized by uncertainty intervals, $D^{*} \pm \mathrm{u}\left(D^{*}\right)$ and $D^{\#} \pm \mathrm{u}\left(D^{\#}\right)$, respectively, to provide safe results. For convenience, however, in the present work, the decision threshold and detection limit were treated as equivalent to their upper limits, $D^{*}+u\left(D^{*}\right)$ and $D^{\#}+u\left(D^{\#}\right)$, respectively. By using the propagation of uncertainties, $u\left(D^{*}\right)$ and $u\left(D^{\#}\right)$ are given by

$$
\mathrm{u}\left(D^{*}\right)=\sqrt{\left(\frac{p^{*}}{a^{2}}\right)^{2} \mathrm{u}(a)^{2}}
$$

and

$$
\mathrm{u}\left(D^{\#}\right)=\sqrt{\left(\frac{p^{\#}}{a^{2}}\right)^{2} \mathrm{u}(a)^{2}}
$$

respectively. The measured peak-to-peak signal of an unknown sample, $p_{s}$, is considered "detected" if $p_{s}>p^{*}$. It is then given a confidence interval with lower limit $p^{\triangleleft}=p_{s}-k_{1-\gamma / 2} u\left(p_{s}\right)$ and upper limit $p^{\triangleright}=p_{s}+k_{1-\gamma / 2} u\left(p_{s}\right)$. If $p_{s}<p^{*}$, the sample cannot be considered "detected" and should be given only the upper limit $\mathrm{p}^{\triangleright}=\mathrm{p}_{\mathrm{s}}+\mathrm{k}_{1-\gamma} \mathrm{u}\left(\mathrm{p}_{\mathrm{s}}\right)$.

The means of the 20 acquired peak-to-peak values on each tablet resulted in 10 values for the background and 5 values for the irradiated tablets. These values were used to calculate the mean and standard deviation of the peak-to-peak values for the background, $p_{0}$ and $u\left(p_{0}\right)$, respectively, as well as those for the irradiated tablets, $p_{1}$ and $u\left(p_{1}\right)$, respectively. The decision threshold, $p^{*}$, was calculated according to (2) with $\alpha=0.05$, but using students coefficient $t_{1-\alpha, 10}=1.81$ for 10 degrees of freedom instead of the standardized normal distribution coefficient $k_{1-\alpha}$. The detection limit, $p^{\#}$, was determined from (3) and (4) by iteration, with $t_{1-\alpha, 5}=2.02$. It was assumed that the uncertainty of the given dose was negligible. The values of $a$ and $P_{0}$ were determined by a least-squares fit of $p_{j}=$ $a D_{j}+P_{0}$, for the 15 data points $\left(D_{j}, p_{j}\right)$, where $D_{j}$ is the given dose given to the jth sample.

The measurement of an unknown sample should be performed analogously with the calibration measurement. Any deviation i from the calibration will contribute with an uncertainty $\mathrm{u}_{\mathrm{i}}\left(\mathrm{p}_{\mathrm{s}}\right)$ to the total uncertainty of the unknown sample $u_{\text {tot }}\left(p_{s}\right)$. The uncertainty from the measurement itself, $u\left(p_{s}\right)$, is obtained from (4). The others are uncertainties from sample fading, $\mathrm{u}_{1}\left(\mathrm{p}_{\mathrm{s}}\right) ;$ storage effects, $\mathrm{u}_{2}\left(\mathrm{p}_{\mathrm{s}}\right)$; irradiation temperature, $\mathrm{u}_{3}\left(\mathrm{p}_{\mathrm{s}}\right)$; and differences in irradiation energy, $u_{4}\left(p_{s}\right)$. The combined total uncertainty is given by $u_{\text {tot }}\left(p_{s}\right)=\sqrt{u\left(p_{s}\right)^{2}+u_{1}\left(p_{s}\right)^{2}+\ldots u_{4}\left(p_{s}\right)^{2}}$.

\section{ACKNOWLEDGEMENTS}

The authors would like to thank Sara Olsson and Alexandru Dasu at the Department of Radiation Treatment for help with sample irradiations and Håkan Pettersson, Emelie Adolfsson and Mattias Karlsson for fruitful discussions.

\section{FUNDING}

This work was supported by the Swedish Civil Contingencies Agency (MSB); and the Swedish Radiation Safety Authority (SSM) [SSI P1519:09, SSM 2010/4164]. H. Gustafsson acknowledges The Swedish Research Council (diarienr 2009-5430) for financial support.

\section{REFERENCES}

1. Alexander, G. A., Swartz, H. M., Amundson, S. A., Blakely, W. F., Buddemeier, B., Gallez, B., Dainiak, N., Goans, R. E., Hayes, R. B., Lowry, P. C. et al. BiodosEPR-2006 Meeting: Acute dosimetry consensus committee recommendations on biodosimetry applications in events involving uses of radiation by terrorists and radiation accidents. Radiation Measurements. 42, 972-996 (2007).

2. Trompier, F., Bassinet, C., Wieser, A., Angelis, C. D., Viscomi, D. and Fattibene, P. Radiationinduced signals analysed by EPR spectrometry applied to fortuitous dosimetry. Annali dell'Istituto Superiore di Sanita. 45, 287-296 (2009).

3. Beerten, K., Woda, C. and Vanhavere, F. Thermoluminescence dosimetry of electronic components from personal objects. Radiation Measurements. 44, 620-625 (2009).

4. Bøtter-Jensen, L. and McKeever, S. W. S. Optically stimulated luminescence dosimetry using natural and synthetic materials. Radiat Prot Dosim. 65, 273-280 (1996).

5. International Commission on Radiation Units and Measurements. Retrospective Assessment of 
Exposure to Ionizing Radiation, ICRU report 68. (Oxford) (2002).

6. International Atomic Energy Agency. Use of electron paramagnetic resonance dosimetry with tooth enamel for retrospective dose assessment, IAEA-TECDOC-1331. IAEA Vienna) (2002).

7. Clairand, I., Trompier, F., Bottollier-Depois, J. F. and Gourmelon, P. Ex vivo ESR measurements associated with Monte Carlo calculations for accident dosimetry: Application to the 2001 Georgian accident. Radiat Prot Dosim. 119, 500505 (2006).

8. Trompier, F., Romanyukha, A., Kornak, L., Calas, C., LeBlanc, B., Mitchell, C., Swartz, H. and Clairand, I. Electron paramagnetic resonance radiation dosimetry in fingernails. Radiation Measurements. 44, 6-10 (2009).

9. Fattibene, P. and Callens, F. EPR dosimetry with tooth enamel: A review. Appl Radiat Isotopes. 68, 2033-2116 (2010).

10. Nakajima, T. ESR of Sugar as a Personnel Monitor for Radiation Emergencies. Appl Radiat Isotopes. 46, 819-825 (1995).

11. Wu, K., Sun, C. P. and Shi, Y. M. Dosimetric Properties of Watch Glass - a Potential Practical Esr Dosimeter for Nuclear Accidents. Radiat Prot Dosim. 59, 223-225 (1995).

12. Trompier, F., Della Monaca, S., Fattibene, P. and Clairand, I. EPR dosimetry of glass substrate of mobile phone LCDs. Radiation Measurements. 46, 827-831 (2011).

13. Hutt, G., Brodski, L. and Polyakov, V. Gamma-ray dose assessment after the 1994 radiation accident in Kiisa (Estonia): Preliminary results. Appl Radiat Isotopes. 47, 1329-1334 (1996).

14. Nakajima, T. Estimation of Absorbed Dose to Evacuees at Pripyat-City Using Esr Measurements of Sugar and Exposure Rate Calculations. Appl Radiat Isotopes. 45, 113-120 (1994).

15. Sagstuen, E., Theisen, H. and Henriksen, T. Dosimetry by ESR spectroscopy following a radiation accident. Health Phys. 45, 961-8 (1983).

16. Shiraishi, K. ESR Dose Estimation of the Radiological Accident in Samut Prakarn, Thailand Using Sugar Samples and an ESR Method. Proceedings of Advances in ESR Applications, 3 (2002).
17. Hervé, M. L., Trompier, F., Tikunov, D. D., Amouroux, V. and Clairand, I. Study of materials for mixed field dosimetry by EPR spectroscopy. Radiat Prot Dosim. 120, 205-209 (2006).

18. Hervé, M. L. Dosimétrie d'accident en champ mixte (neutrons, photons) utilisant la spectrométrie par résonance paramagnétique électronique (RPE). (Universite Paris XI) (2006).

19. Kinoshita, A., Jose, F. A. and Baffa, O. An Attempt to Use Sweeteners as a Material for Accident Dosimetry. Health Physics. 98, 406-411 (2010).

20. Budzinski, E. E., Potter, W. R. and Box, H. C. Radiation Effects in X-Irradiated Hydroxy Compounds. J Chem Phys. 72, 972-975 (1980).

21. Vanhaelewyn, G., Sadlo, J., Callens, F., Mondelaers, W., De Frenne, D. and Matthys, P. A decomposition study of the EPR spectrum of irradiated sucrose. Appl Radiat Isotopes. 52, 12211227 (2000).

22. International Organization for Standardization. Determination of the Characteristic Limits (Decision Threshold, Detection Limit and Limits of the Confidence Interval) for Measurements of Ionizing Radiation - Fundamentals and Application, ISO 11929 ((Geneva:ISO)) (2010).

23. Currie, L. A. Limits for qualitative detection and quantitative determination: Application to radiochemistry. Anal Chem. 40, 586-593 (1968).

24. Maghraby, A. and Salama, E. Use of aspartamebased sweetener tablets in emergency dosimetry using EPR. Radiat Prot Dosim. 139, 505-509 (2010).

25. Nagy, V. Accuracy considerations in EPR dosimetry. Appl Radiat Isotopes. 52, 1039-1050 (2000). 
Table 1. Table describing the different experiments.

\begin{tabular}{|c|c|c|c|c|c|c|c|c|c|c|}
\hline Experiment & $\begin{array}{l}\text { Number } \\
\text { of } \\
\text { tablets }^{\mathrm{a}}\end{array}$ & Mass (mg) & Dose (Gy) & $\begin{array}{l}\text { Time since } \\
\text { irradiation (days) }\end{array}$ & $\begin{array}{l}\text { Spectrometer } \\
\text { used }\end{array}$ & $\begin{array}{l}\text { Microwave } \\
\text { power } \\
(\mathrm{mW})\end{array}$ & $\begin{array}{l}\text { Modulation } \\
\text { amplitude } \\
(\mathrm{mT})\end{array}$ & $\begin{array}{l}\text { Number of } \\
20 \mathrm{~s} \\
\text { sweeps }\end{array}$ & $\begin{array}{l}\text { Time } \\
\text { constant } \\
(\mathrm{ms})\end{array}$ & $\begin{array}{l}\text { Conversion } \\
\text { time }(\mathrm{ms})\end{array}$ \\
\hline $\begin{array}{c}\text { Signal evolution with } \\
\text { time }\end{array}$ & 1 & 200 & 50 & $1,4,8,16,36,84$ & Bruker & 1 & 0.2 & $80^{\mathrm{b}}$ & 10 & 20.5 \\
\hline Power saturation & 1 & 200 & 50 & $1,16,84$ & Bruker & $0.2-100^{c}$ & 0.2 & $8^{\mathrm{d}}$ & 10 & 20.5 \\
\hline $\begin{array}{l}\text { Effects of storage } \\
\text { conditions }\end{array}$ & 6 & 200 & 10 & 69 & Bruker & 100 & 0.1 & $10^{\mathrm{e}}$ & 1.28 & 20.5 \\
\hline $\begin{array}{l}\text { Linearity of the dose } \\
\text { response }\end{array}$ & 6 & $98-102$ & $\begin{array}{c}0.5,1,3,5 \\
10,20\end{array}$ & 4 & JEOL & 4 & 0.5 & $100^{\mathrm{e}}$ & 10 & 4.9 \\
\hline $\begin{array}{c}\text { Dose response } \\
\text { measurement }\end{array}$ & 10 & $96-100$ & 4 & $4-6$ & JEOL & 4 & 0.5 & $400^{\mathrm{e}}$ & 10 & 4.9 \\
\hline
\end{tabular}

${ }^{a}$ per sample type

per measurement occasion

c 10 different powers ramped up exponentially

${ }^{\mathrm{d}}$ per microwave power

per tablet and dose 
Table 1: The results of the blind test. The uncertainty of the given dose is neglected. The uncertainty of the measured dose is given as one standard deviation (SD). It can be seen that, for all samples except one, the measured dose coincides with the given dose within the uncertainty of $1 \mathrm{SD}$.

\begin{tabular}{rccc}
\hline Sample & Given dose (Gy) & Measured dose (Gy) & Absolute Difference (Gy) \\
\hline A & 0.85 & $0.74 \pm 0.17$ & 0.11 \\
B & 0.88 & $0.95 \pm 0.17$ & 0.07 \\
C & 2.97 & $2.81 \pm 0.15$ & 0.17 \\
D & 3.05 & $3.15 \pm 0.14$ & 0.10 \\
\hline
\end{tabular}

